\title{
EQUILIBRIO HIDRÁULICO EN SISTEMAS DE BOMBEO MINERO: ESTUDIO DE CASO
}

\section{HYDRAULIC BALANCE ON MINE PUMPING SYSTEMS: A CASE STUDY}

\author{
Luis Enrique Ortiz Vidal ${ }^{1} \quad$ Danny Eugenio Cabanillas Maury $^{2} \quad$ Roger Abel Fierro Chipana $^{2}$
}

Recibido 29 de abril de 2009, aceptado 8 de octubre de 2010

Received: April 29, 2009 Accepted: October 8, 2010

\begin{abstract}
RESUMEN
Fue evaluada la influencia del uso de los métodos de Hazen-Williams y Darcy-Weisbach en el establecimiento del equilibrio hidráulico para un sistema de bombeo minero. Empresas mineras con actividad subterránea hacen uso de estaciones de bombeo para evacuar el agua, producto de la profundización de sus labores. Proyectistas y vendedores de equipos de bombeo usan diferentes expresiones para la estimación de la pérdida de carga total del sistema, parámetro importante para la determinación del equilibrio hidráulico. El presente estudio tiene como objetivo analizar y validar la aplicación de algunas de estas expresiones para un sistema de bombeo minero. Las principales características del estudio de caso son: caudal de agua de 1.350 1/s; tuberías de acero y HDPE de 16" y 18" de diámetro, respectivamente; longitud total de la tubería de $2.900 \mathrm{~m}$ y una altura geodésica de $230 \mathrm{~m}$. Los cálculos fueron realizados con los métodos ya mencionados teniendo las expresiones de Haaland, Swamee-Jain y Churchill como factores de fricción. Los resultados obtenidos fueron comparados con los medidos en campo, teniéndose una desviación máxima del sistema de $28,6 \%$ y $3,1 \%$ para la pérdida de carga y Hman total, respectivamente.
\end{abstract}

Palabras clave: Bombeo minero, equilibrio hidráulico, flujo en tuberías, pérdida de carga, factor de fricción.

\section{ABSTRACT}

This study evaluates the influence of the Hazen-Williams and Darcy-Weisbach methods on the hydraulic balance of a mine pumping system. Underground mining companies use pumping stations for evacuate the produced water. Designers and equipment sellers use different expressions to estimate the head loss. This study analyzes and validates the implementation of some of these expressions to a mine pumping system. The features of the case study are: water flow rate of $1350 \mathrm{l} / \mathrm{s}$, steel and HDPE diameter pipes of $16 \mathrm{in}$. and $18 \mathrm{in}$., respectively. The total pipe length is $2900 \mathrm{~m}$, and the hydraulic height difference is $230 \mathrm{~m}$. The calculations were performed by the above-mention methods, taking the expressions of Haaland, Swamee-Jain and Churchill for friction factors. The results were compared with those measured in field, taking a maximum deviation of system of $28.6 \%$ and $3.1 \%$ for head loss and total head, respectively.

Keywords: Mining pumping, hydraulic balance, pipes flow, head loss, friction factor.

\section{INTRODUCCIÓN}

Sistemas de bombeo están presentes en la industria minera con actividad subterránea cuando el agua de mina, producto de las excavaciones, debe ser bombeada hacia superficie para garantizar la continuidad de la producción. La selección de estos sistemas de bombeo precisa del correcto establecimiento del equilibrio hidráulico del sistema. Ingenieros proyectistas y vendedores hacen uso de las ecuaciones de conservación de la masa, energía y cantidad de movimiento para esta actividad. Errores al establecer el equilibrio hidráulico ocasionan graves consecuencias en la estación de bombeo. Es decir, al sobredimensionar un sistema se tendrían equipos más potentes trabajando a menores eficiencias ocasionando pérdidas económicas y técnicas; en el caso contrario se tendrían sistemas sin capacidad suficiente para evacuar el agua requerida.

En sistemas de bombeo, el equilibrio hidráulico es obtenido a partir de un balance de energía, donde son contabilizadas la energía cinética, energía potencial y pérdidas de energía. En este sentido, un equívoco bastante difundido entre profesionales, que se dedican al dimensionamiento

\footnotetext{
1 Núcleo de Engenharia Térmica e Fluidos (NETeF). Escola de Engenharia de São Carlos. Universidade de São Paulo. Av. Trabalhador São-carlense, 400, 13566-970. São Carlos-SP, Brasil. E-mail: leortiz@ pucp.pe; leortiz@sc.usp.br

2 Departamento de Ingeniería y Proyectos. Consultora Minera Proyectos y Estudios Mineros S.A.C. Calle Fray Angélico 238 San Borja. Lima, Perú. E-mail: dcabanillas@pucp.edu.pe; rogerfit@gmail.com
} 
de sistemas de bombeo, radica en llamar de "ecuación de Bernoulli con pérdidas" a la ecuación de energía. La ecuación de Bernoulli fue deducida para flujos sin pérdidas de energía [1-2]. Expresiones como "Bernoulli con pérdidas" o "Bernoulli modificada" solo pertenecen al argot cotidiano.

Las pérdidas de energía se deben principalmente a turbulencia y fricción. Estas han sido ampliamente estudiadas y contabilizadas en la pérdida de carga total del sistema. Los métodos de Darcy-Weisbach y HazenWilliams son los más aplicados para el cálculo de pérdida de carga en tuberías, teniéndose el segundo método como el más usado en el sector industrial y el primero como el más preciso. A pesar de la mejor precisión de DarcyWeisbach, el método deja de ser aplicado por la aparente complejidad en el cálculo del factor de fricción [3].

La primera ecuación para el cálculo del factor de fricción (f) en tuberías comerciales fue dada en 1939 por Cyril F. Colebrook. En su afán de entender las transiciones de flujo observadas por Nikuradse, Colebrook desarrolló una expresión para el cálculo de factor de fricción que vincula el número de Reynolds $(R e)$ y la rugosidad relativa de la tubería $(e / D)[4]$ :

$$
\frac{1}{\sqrt{f}}=-2,0 \log \left(\frac{e / D}{3,7}+\frac{2,51}{\operatorname{Re} \sqrt{f}}\right)
$$

La ecuación (1), comúnmente llamada ecuación de Colebrook, no fue atractiva para muchos ingenieros debido a su carácter implícito y a la ausencia de recursos de cálculo en la época. Fue entonces que en 1942 el ingeniero Hunter Rouse tabuló los resultados de la ecuación de Colebrook para hacer más fácil el cálculo del factor de fricción. El trabajo de Hunter Rouse fue conocido-dos años después-a través de Lewis F. Moody, quien presentó sus resultados en el que hoy se conoce como Diagrama de Moody. Desde entonces el desarrollo científico -en este sentido- fue volcado hacia explicitar la ecuación de Colebrook, teniendo a Swamee-Jain (1976), Churchill (1977) y Haaland (1983) como las expresiones más representativas para el cálculo del factor de fricción $[1,3]$.

El método de Hazen-Williams para el cálculo de pérdidas de carga total ofrece ventajas de cálculo frente a Darcy-Weisbach. Sin embargo, aun cuando este método es ampliamente aceptado en ingeniería, no tiene en cuenta fenómenos físicos inherentes al proceso de fricción y turbulencia. No obstante, tomando las consideraciones necesarias, el uso del método de Hazen-Williams se hace válido en muchas aplicaciones de ingeniería como en el presente estudio de caso.
Analizar y validar los métodos de Hazen-Williams y Darcy-Weisbach con Haaland, Swamee-Jain y Churchill como expresiones de factor de fricción en un estudio de caso son los objetivos del presente trabajo.

Los resultados obtenidos muestran una variación máxima del 28,6\% para el cálculo de pérdida de carga total entre los diferentes métodos y expresiones. Sin embargo, para el caso de sistemas de bombeo mineros con características similares a las presentadas, se concluye que la elección del método de cálculo no tiene relevancia global sobre el equilibrio hidráulico del sistema.

\section{DESCRIPCIÓN DEL SISTEMA DE BOMBEO}

La Figura 1 muestra el esquema del sistema de bombeo referente al estudio de caso. Las letras arábigas representan las cinco partes del sistema, las que están listadas en la Tabla 1. La Tabla 2 lista los elementos de seguridad y regulación de cada línea de bombeo.

Tabla 1. Etapas del sistema de bombeo.

\begin{tabular}{|c|l|}
\hline Ítem & \multicolumn{1}{|c|}{ Descripción } \\
\hline A & Poza y canal de captación \\
\hline B & Desarenador \\
\hline C & Poza de succión \\
\hline D & Estación de bombeo \\
\hline E & Poza de descarga \\
\hline
\end{tabular}

Tabla 2. Accesorios de la estación de bombeo.

\begin{tabular}{|l|l|}
\hline Item & \multicolumn{1}{|c|}{ Descripción } \\
\hline \\
\hline
\end{tabular}

El agua producto de la profundización de labores mineras es dirigida - por gravedad o por bombeo- y captada en la poza A. Un canal de captación con pendiente negativa de $1 \%$ deriva el agua hacia el desarenador $\mathrm{B}$, donde el 
ingreso del agua es controlado mediante compuertas metálicas. Las dimensiones del desarenador son tales que permiten la sedimentación de sólidos gruesos (partículas con diámetro mayor de $2,5 \mathrm{~mm}$ ) y reducir el porcentaje de sólidos finos en suspensión de 250 ppm a 50 ppm. El agua desarenada es almacenada en la poza de succión C. Además de servir de almacenamiento, la poza de succión $\mathrm{C}$ trabaja como un amortiguador natural -colchón de agua- para el sistema de bombeo con succión positiva, ayudando a tener una alimentación constante y manteniendo el NPSH en equilibrio. La estación de bombeo D traslada el agua almacenada por medio de cinco bombas centrífugas de
$1.500 \mathrm{HP}$ y 270 1/s cada una. Un sistema de alivio en caso ocurra un golpe de ariete está presente en la estación de bombeo. Una válvula anticipadora de onda acciona el sistema a partir de cualquier corte de energía o paro repentino de la bomba. Como contingencia ante un mal funcionamiento del sistema de alivio existen discos de rupturas que actuarían como absorbedores de energía originadas por el golpe de ariete. La Figura 1 muestra el sistema de tuberías por donde fluye el agua, su longitud es de $2.955 \mathrm{~m}$ incluyendo los $230 \mathrm{~m}$ de altura geodésica. En superficie -"Nivel Superior" en la Figura 1- el agua alcanza la poza de descarga $\mathrm{E}$.

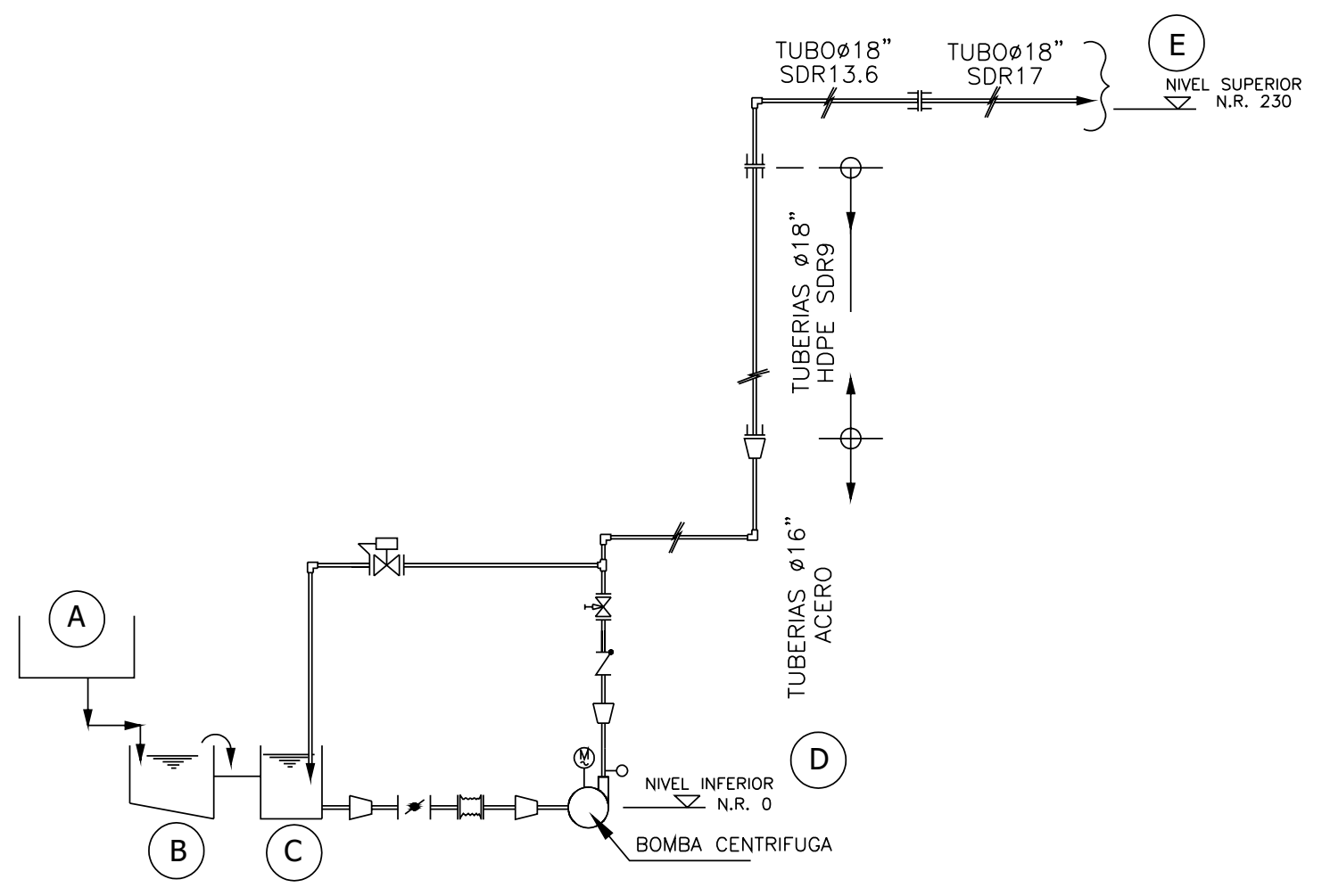

Figura 1. Esquema del sistema de bombeo.

\section{ECUACIONES GOBERNANTES}

El equilibrio hidráulico es establecido a partir de las ecuaciones de conservación de la masa, cantidad de movimiento y energía. Estas ecuaciones son sometidas a un conjunto de hipótesis simplificadoras con la finalidad de representar adecuadamente el fenómeno físico. La primera hipótesis a ser tomada es considerar al transporte de agua como un flujo monofásico. Esta asunción es válida y lícita ya que el agua de mina -producto de las excavaciones- pasa a través de estructuras hidráulicas correctamente diseñadas que reducen el porcentaje de sólidos a valores cercanos a cero [5].
Ecuación de conservación de la masa para volúmenes de control

$$
0=\frac{\partial}{\partial t} \int_{\forall . c .} \rho d \forall+\int_{\text {s.c. }} \rho \vec{V} d \vec{A}
$$

siendo $\rho$ la densidad del fluido, $V$ la velocidad del fluido; "v.c." y "s.c." los subíndices que indican volumen y superficie de control, respectivamente.

La ecuación integral básica para conservación de la masa representada por la ecuación (2) es simplificada para el 
transporte de fluidos en tuberías. Tomando las hipótesis de régimen permanente, volumen de control fijo, flujo uniforme, sección constante, una entrada-una salida y flujo incompresible, la ecuación de conservación de la masa adquiere la siguiente forma [1-2,6]

$$
0=\dot{m}_{e}-\dot{m}_{s}
$$

Ecuación de energía para volúmenes de control

$$
\dot{Q}-\dot{W}=\frac{\partial}{\partial t} \int_{\forall . c .} e \rho d \forall+\int_{s . c .}\left(h+\frac{V^{2}}{2}+g z\right) \rho \vec{V} \cdot d \vec{A}
$$

siendo $Q$ el flujo de calor en el volumen de control; $W$ la suma de trabajos, entre otros, trabajo de eje y tensiones tangenciales, transmitidos a través de las superficies de control; $e$ la energía específica; $h$ la entalpía; $g$ aceleración de la gravedad; $z$ la diferencia de cotas. Los subíndices "v.c." y "s.c." indican volumen y superficie de control, respectivamente.

La ecuación de la energía representada en su forma integral por la ecuación (4) es reducida y acoplada a la ecuación simplificada de conservación de la masa simplificada. La ecuación (5) muestra el acople tomando las hipótesis de régimen turbulento, permanente e incompresible, ausencia de trabajo y energía y presión uniformes en las secciones de entrada y salida $[1-2,6]$

$$
\left(\frac{P_{e}}{\rho g}+\frac{V_{e}^{2}}{2 g}+Z_{e}\right)-\left(\frac{P_{s}}{\rho g}+\frac{V_{s}^{2}}{2 g}+Z_{s}\right)=h_{l T}
$$

siendo $P$ presión $(\mathrm{Pa}) ; \mathrm{V}$ velocidad media $(\mathrm{m} / \mathrm{s}) ; \mathrm{Z}$ altura manométrica $(m) ; h_{l T}$ pérdida de carga total $(m)$. Los subíndices " $e$ " y " $s$ " indican entrada y salida, respectivamente.

\section{PÉRDIDA DE CARGA}

Ecuaciones constitutivas o de acople son necesarias para el establecimiento del equilibrio hidráulico. En el caso de transporte de fluidos en tuberías, estas contabilizan la pérdida de carga por efecto -principalmente- de la fricción y turbulencia. Las pérdidas de carga $\left(h_{l}\right)$ son de tipo primarias o distribuidas $\left(h_{l p}\right)$ y secundarias o localizadas $\left(h_{l s}\right)$, la suma de estas es conocida como pérdida de carga total $\left(h_{l T}\right)$ [6-7].

\section{Método de Darcy-Weisbach [1-2,6]}

Darcy-Weisbach es un método utilizado en el cálculo de pérdida de carga; su naturaleza semiempírica hace que pueda ser aplicado para diferentes tipos de flujo, fluidos y tuberías. Las pérdidas de carga primarias $\left(h_{l p}\right)$ y secundarias $\left(h_{l s}\right)$ pueden calcularse mediante

$$
\begin{aligned}
& h_{l p}=f\left(\frac{L}{D}\right) \frac{V^{2}}{2} \\
& h_{l s}=f\left(\frac{L_{e}}{D}\right) \frac{V^{2}}{2}
\end{aligned}
$$

siendo $f$ el factor de fricción de Darcy, $L$ la longitud de tubería $(m), L_{e}$ la longitud equivalente de accesorios obtenido de tablas $(m), D$ el diámetro de tubería $(m) ; V$ la velocidad media del fluido.

Las ecuaciones (8), (9) y (10-12) muestran las expresiones explícitas desarrolladas por Swamee-Jain, Haaland y Churchill, respectivamente, para el cálculo del factor de fricción de Darcy, cada una de ellas en función del número adimensional de Reynolds $(R e)$ y la rugosidad relativa de la tubería $(e / D)[1,4,8]$.

Swamee-Jain (1976): válida para régimen turbulento totalmente desarrollado,

$$
f=1,325\left\{\ln \left[0,27\left(\frac{e}{D}\right)+5,74\left(\frac{1}{\mathrm{Re}}\right)^{0,9}\right]\right\}^{-2}
$$

Haaland (1983): válida para régimen de transición y turbulento,

$$
f=\left\{-1,8 \log \left[\frac{6,9}{\mathrm{Re}}+\left(\frac{e / D}{3,7}\right)^{1,11}\right]\right\}^{-2}
$$

Churchill (1977): válida para todos los padrones de flujo,

$$
f=8\left[\left(\frac{8}{\operatorname{Re}}\right)^{12}+\frac{1}{(A+B)^{3 / 2}}\right]^{1 / 12}
$$

$$
\begin{gathered}
A=\left\{2,457 \ln \left[\frac{1}{\left(\frac{7}{\mathrm{Re}}\right)^{0,9}+0,27\left(\frac{e}{D}\right)}\right]\right\}^{16} \\
B=\left[\frac{37530}{\mathrm{Re}}\right]^{16}
\end{gathered}
$$

\section{Método de Hazen-Williams [7]}

Hazen-Williams es un método de cálculo de pérdida de carga en tuberías; su naturaleza empírica hace que su aplicación sea restringida al transporte de agua bajo ciertas condiciones [9]. Las pérdidas de carga primarias $\left(h_{l p}\right)$ y secundarias $\left(h_{l s}\right)$ pueden calcularse mediante 


$$
\begin{gathered}
h_{l p}=10,643 L\left(\frac{Q}{C}\right)^{1,85} D^{-4,87} \\
h_{l s}=K \frac{V^{2}}{2}
\end{gathered}
$$

siendo $C$ el coeficiente de Hazen-Williams, $Q$ el caudal de agua $\left(\mathrm{m}^{3} / \mathrm{s}\right), K$ la constante de accesorios obtenido de tablas $[1-2,6]$.

El equilibrio hidráulico es establecido cuando es resuelta la ecuación (5) haciendo uso de alguno de los dos métodos presentados.

\section{RESULTADOS Y DISCUSIÓN}

Fueron comparados los métodos de Hazen-Williams y Darcy-Weisbach con Haaland, Churchill y Swamee-
Jain como expresiones para el factor de fricción, para el establecimiento del equilibrio hidráulico, siendo las condiciones del sistema las descritas anteriormente. El sistema de bombeo estudiado presenta un número de Reynolds entre $8,8 \times 10^{5}$ y $9,7 \times 10^{5}$.

Las expresiones de Swamee-Jain, Haaland y Churchill fueron implementadas para tuberías de acero Ø16" y HDPE $\varnothing 18$ " con la finalidad de observar su comportamiento en tuberías rugosas y lisas, respectivamente. Los resultados son mostrados en las Figuras 2 y 3 . Se nota que en el caso de tuberías rugosas, las expresiones de Swamee-Jain y Churchill se superponen, mientras que la expresión de Haaland prevé valores menores para el coeficiente de fricción. Un comportamiento diferente es observado para tuberías lisas; las tres expresiones presentan valores casi similares. Este resultado muestra que es irrelevante la elección de cualquiera de estas expresiones para el cálculo de pérdida de carga en tuberías lisas.

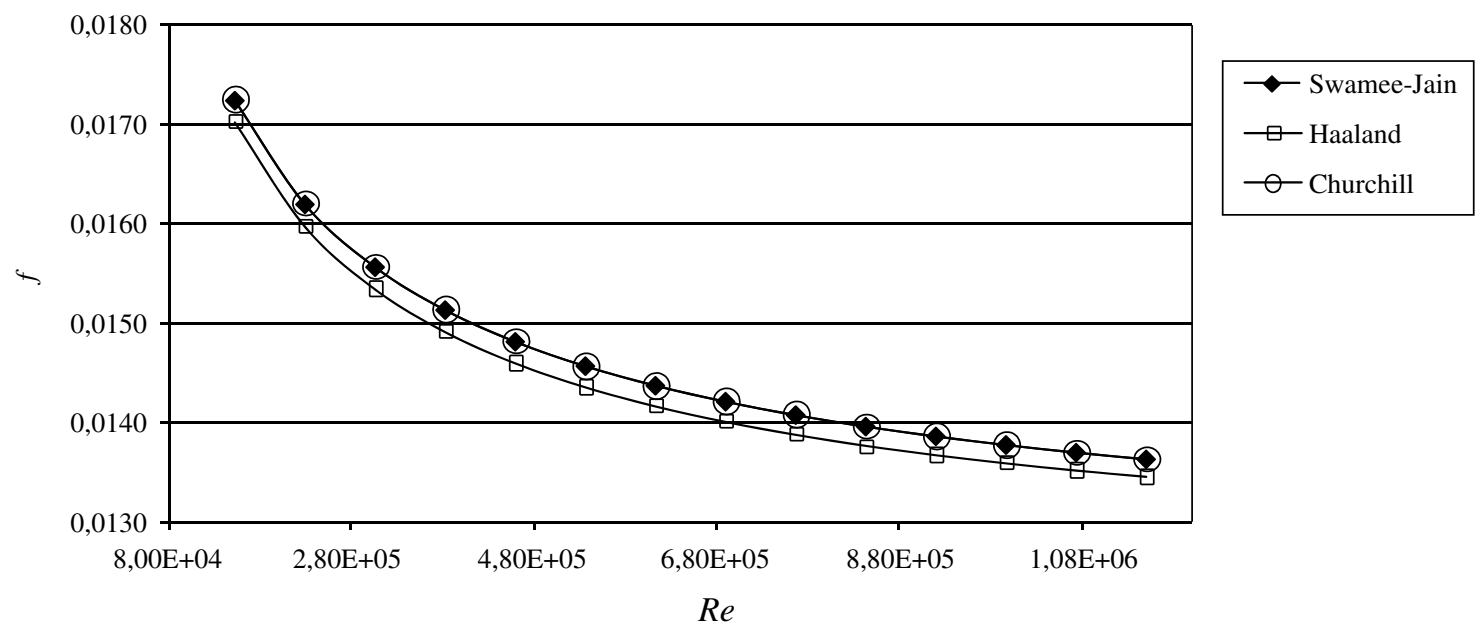

Figura 2. Factor de fricción en tubería rugosa en función de Re. Tubo de acero $\emptyset 16^{\prime \prime}\left(e / D=1,19 \times 10^{-4}\right)$.

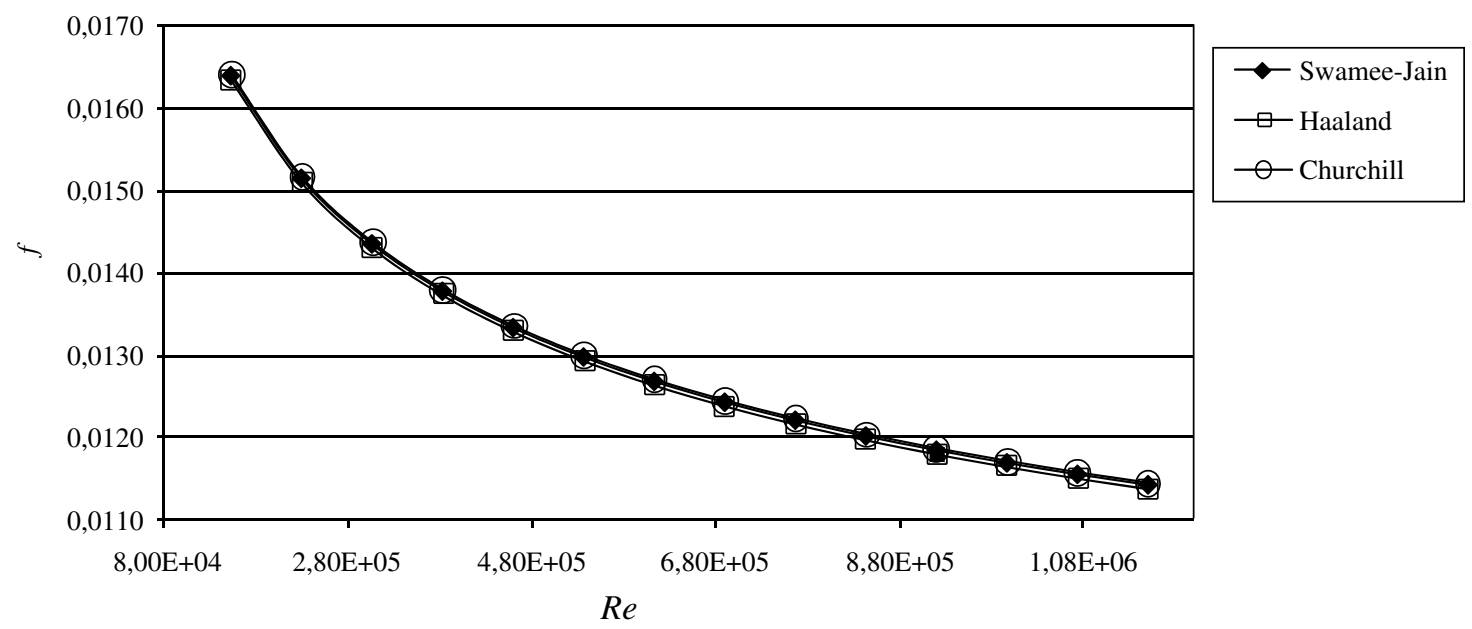

Figura 3. Factor de fricción en tubería lisa en función de Re. Tubo de HDPE $\emptyset 18 ”\left(\mathrm{k} / \mathrm{D}=3,87 \times 10^{-6}\right)$. 
El comportamiento de los métodos Darcy-Weisbach y Hazen-Williams fue comparado para tuberías rugosas y lisas de acero Ø16” y HDPE Ø18”, respectivamente. Para el método de Darcy-Weisbach fueron implementadas solo las expresiones de Haaland y Churchill. Resultados de pérdida de carga por kilómetro de tubería en función del número de Reynolds ( $R e$ ) son mostrados en las Figuras 4 y 5. Para ambos casos, tuberías rugosas y lisas, se observa que el método de Darcy-Weisbach prevé menores pérdidas de carga que el método de Hazen-Williams, haciéndose más notorio en tuberías rugosas. La diferencia se incrementa con el aumento en el número de Reynolds.

En sistemas de bombeo minero no son encontrados sistemas de tuberías de acero de grandes longitudes, por este motivo los resultados para tubería lisa, mostrados en la Figura 5, son más representativos en el sentido práctico.

La Figura 6 muestra la pérdida de carga teórica en el sistema de bombeo del presente estudio de caso. Son mostradas las pérdidas primarias, secundarias y totales para cada sección del sistema de tuberías, esto es, para el tramo de acero, HDPE SRD9, SDR13,6 y SDR17. El tramo correspondiente a la tubería de acero presenta altas pérdidas de carga secundarias debido a la presencia de accesorios como válvulas, codos y reducciones. Para la tubería HDPE SDR17, los altos valores para la pérdida de carga primaria son causados por la gran longitud de tubería.

Datos experimentales fueron levantados en campo. Un manómetro ubicado a la salida de la bomba arrojó una lectura promedio de 360 psi. Substituyendo este valor en la ecuación de la energía -ecuación (5)- para las condiciones del estudio de caso fue obtenida la pérdida de carga experimental del sistema. Los resultados de las comparaciones teórico-experimentales para los métodos de Hazen-Williams y Darcy-Weisbach son mostrados en la Figura 7. La desviación teórico-experimental es calculada por el cociente entre la diferencia de medida teórica y

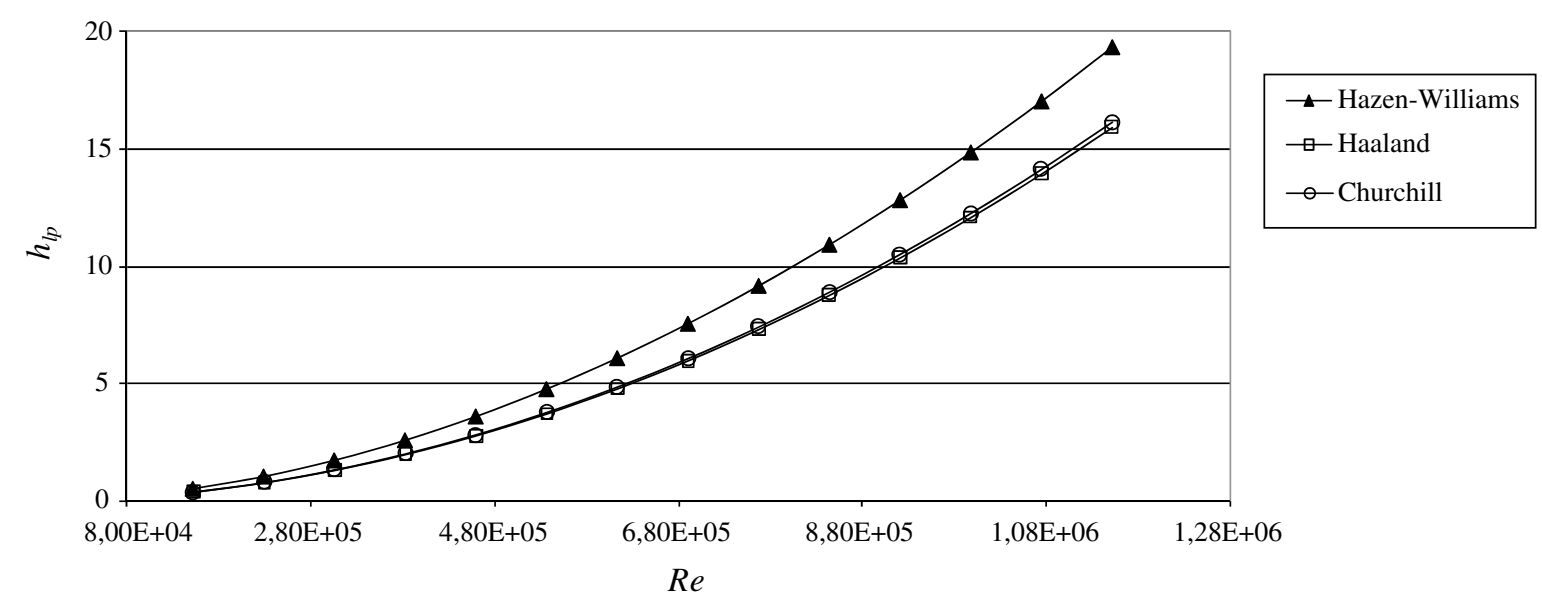

Figura 4. Pérdida de carga por kilómetro de tubería rugosa en función de $R e$. Tubo de acero $\varnothing 16^{\prime \prime}\left(e / D=1,19 \times 10^{-4}\right)$.

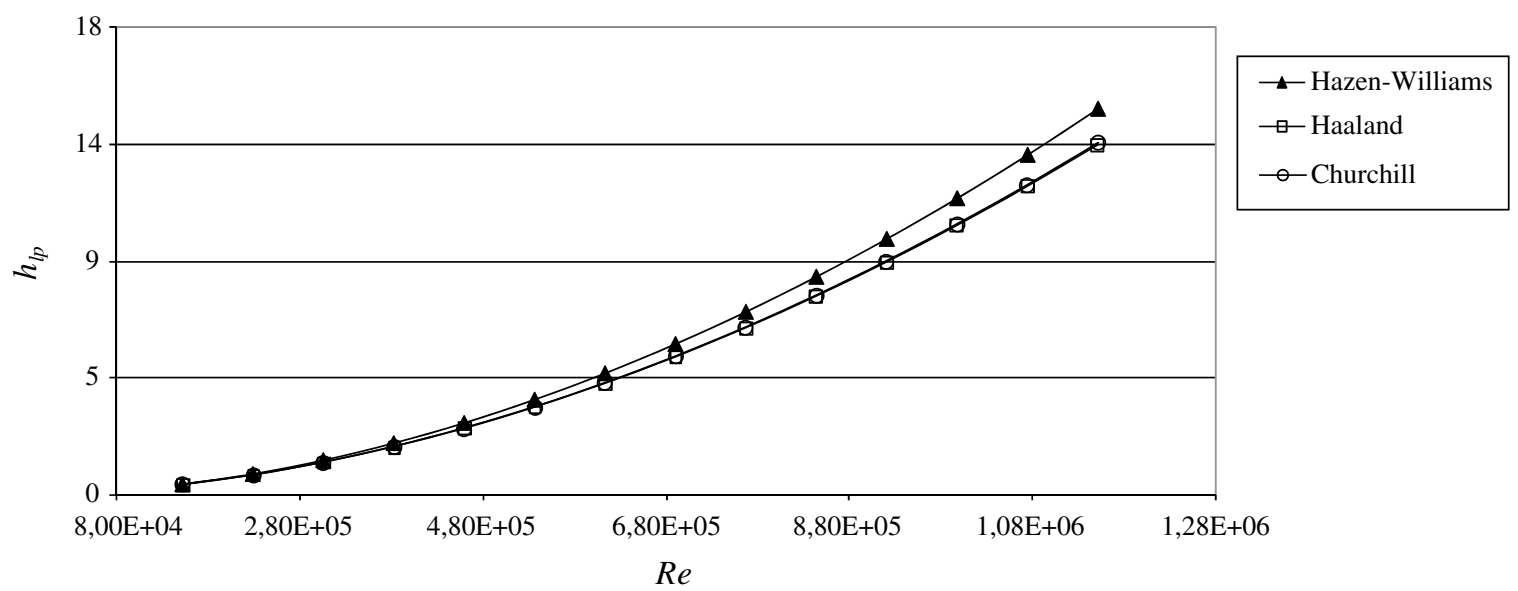

Figura 5. Pérdida de carga por kilómetro de tubería lisa en función de Re. Tubo de HDPE $\varnothing 18^{\prime}\left(\mathrm{k} / \mathrm{D}=3,87 \times 10^{-6}\right)$. 
experimental $\left(M_{t e o}-M_{\text {exp }}\right)$ y la medida experimental $\left(M_{\text {exp }}\right)$. Se observa que el método de Darcy-Weisbach-Churchill prevé menor desviación teórico-experimental, siendo el más preciso con $6,8 \%$. El método de Hazen-Williams fue el más impreciso con una desviación de $28,6 \%$. A pesar del amplio margen de desviación teórico-experimental entre los dos métodos, al compararlos con respecto al Hman total del sistema se obtuvo una desviación de $3,1 \%$ y $1,1 \%$ para Hazen-Williams y Darcy-Weisbach-
Churchill, respectivamente. Estos resultados se deben a que el Hman total incorpora la altura manométrica sistema, siendo esta última más preponderante que la pérdida de carga total.

Debido a que el parámetro usado para la selección de equipos de bombeo es el Hman del sistema, el establecimiento del equilibrio hidráulico puede hacerse con cualquiera de los métodos presentados.

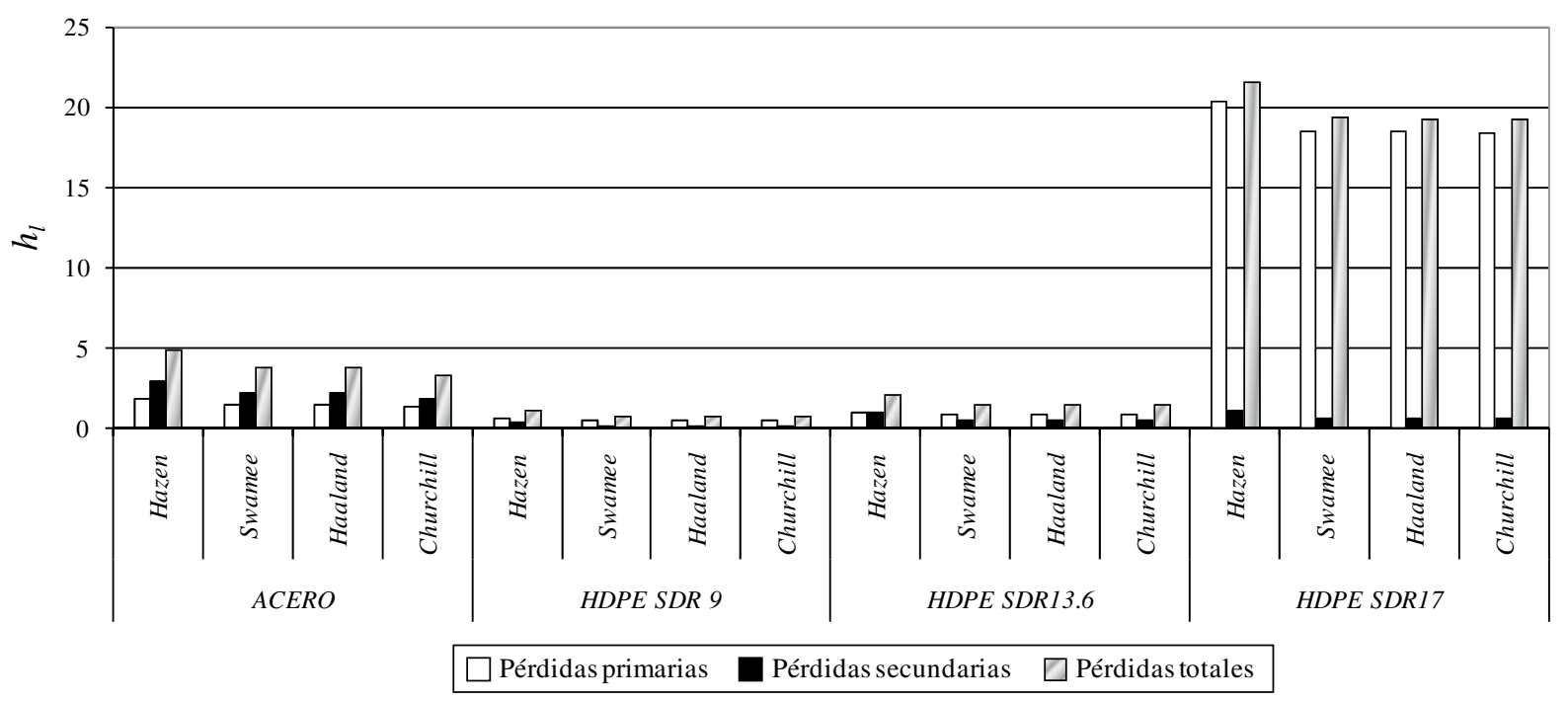

Figura 6. Pérdida de carga teórica en el sistema de bombeo.

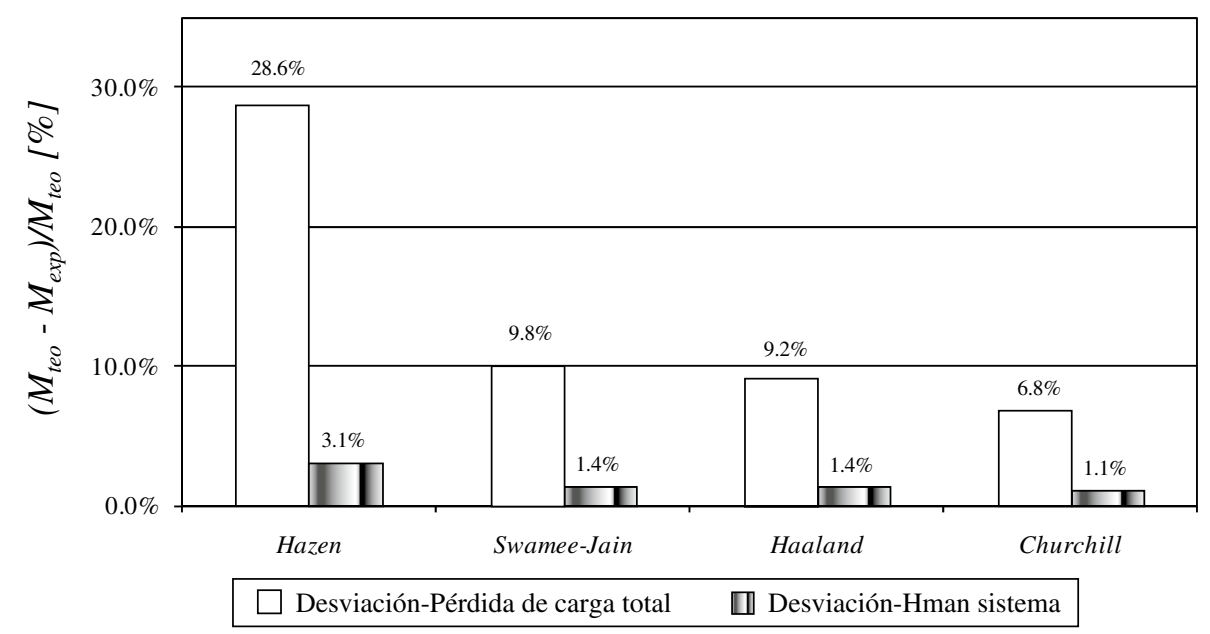

Figura 7. Desviación de la pérdida de carga y Hman total del sistema frente a los datos experimentales. 


\section{CONCLUSIONES}

Fue evaluada la influencia del uso de los métodos de HazenWilliams y de Darcy-Weisbach en el establecimiento del equilibrio hidráulico para un sistema de bombeo minero. Haciendo uso de hipótesis pertinentes, las ecuaciones de continuidad y energía fueron simplificadas y aplicadas a las condiciones del estudio de caso. Las expresiones de Swamee-Jain, Haaland y Churchill para el cálculo del factor de fricción de Darcy fueron comparadas. Previsiones teóricas para pérdida de carga en tuberías rugosas y lisas fueron realizadas para ambos modelos. Los resultados teóricos muestran que el método de Darcy-Weisbach prevé menor pérdida de carga que el método de Hazen-Williams, alcanzando su menor valor con Churchill. Comparaciones teórico-experimentales fueron realizadas para pérdida de carga y Hman del sistema, obteniéndose que el método Darcy-WeisbachChurchill presenta mejor concordancia experimental. Los bajos valores de desviación teórico-experimental de ambos métodos respecto al Hman del sistema indican que cualquiera de ambos métodos puede usarse para el establecimiento del equilibrio hidráulico en un sistema de bombeo minero con las características del presente estudio de caso.

\section{AGRADECIMIENTOS}

Agradecemos a la empresa consultora Proyectos y Estudios Mineros S.A.C. por el apoyo en la construcción de este documento.

\section{REFERENCIAS}

[1] Y.A. Çengel e J.M. Cimbala. "Mecânica dos Fluidos-Fundamentos e Aplicações". McGrawHill, $1^{\text {a }}$ Edição. São Paulo, Brasil. 2007.

[2] R.W Fox, A.L. McDonald e P.J. Pritchard. "Introdução à Mecânica dos Fluidos". LTC-Livros Técnicos e Científicos Editora S.A. Sexta edição. Rio de Janeiro, Brasil. 2006.

[3] M.C. Potter e D.C. Wiggert. "Mecânica dos fluidos". Pionera Thomson Learning. Traducción de la Tercera Edición Norte-Americana. São Paulo, Brasil. 2004.

[4] J.A.A. Sá Marques, J.O.O. Sousa. "Fórmula de Colebrook-White: Velha mas actual. Soluções Explícitas". Fecha de consulta: 8 de octubre de 2010. URL: http://www.hidrotec.xpg.com.br/ FormCole.htm

[5] O.M.H. Rodríguez. "Tópicos Avançados em Mecânica de Fluidos: Modelagem de Escoamento Bifásico em Tubulações, Apostila SEM5872”. Escola de Engenharia de São Carlos-Universidade de São Paulo. São Carlos-SP, Brasil. 2008.

[6] O.M.H. Rodríguez. "Mecânica dos FluidosDisciplina de Pos-Graduação, Apostila SEM5749”. Escola de Engenharia de São Carlos-Universidade de São Paulo. São Carlos-SP, Brasil. 2008.

[7] C. Mataix. "Mecánica de Fluidos y Máquinas hidráulicas". Ediciones del Castillo. Segunda Edición. Madrid, España. 1986.

[8] R.G. Allen. "Relating the Hazen-Williams and DarcyWeisbach friction loss equations for pressurized irrigation". American Society of Agricultural and Biological Engineers. Vol. 12, Issue 6, pp. 685-693. 1996.

[9] C.P. Liou. "Limitations and proper use of the Hazen-Williams Equation". Journal of Hydraulic Engineering. Vol. 124, Issue 9, pp. 951-954. September 1998. 